\title{
Seasonal variations in the process of care in urban general practice
}

\author{
L HALLAM AND D H H METCALFE \\ From the DHSS Primary Care Research Unit, Department of General Practice, University of Manchester
}

SUMMARY Using data collected for a study of the process of care in a large urban area, the opportunity was taken to examine seasonal variations in consultation patterns. While overall numbers of consultations varied month by month, no seasonal pattern was found. The ratio between patient and doctor-initiated contacts remained stable throughout the year, as did the age, sex and social class of patients consulting. The proportion of consultations that took place in the home increased during the winter months. As expected, seasonal fluctuations in morbidity were most apparent in respiratory disorders, though a small number of non-respiratory problems showed similar variations. There was no discernible seasonal pattern to investigation, referral, and prescribing rates, though wide variation existed between individual general practitioners. The implications of these findings for the design of future studies are discussed.

It has long been accepted that morbidity patterns in general practice vary seasonally. From this has followed the belief that the pattern of care will also exhibit seasonal variations, in direct response to the level and type of morbidity presenting. This convention has had serious implications for study design in health services research. The emphasis placed on securing data from at least one full 12-month period often imposes constraints in other areas. For example, the range of information sought may be curtailed to offset the burden of long term recording, and response rates may be low because of the protracted nature of the research.

Accepting conventional wisdom, a major study of the process of care in Greater Manchester, undertaken in 1981/82, was designed to obtain data which accurately represented not only seasons but also days of the week. Participating general practitioners were provided with individual recording schedules, and when it subsequently proved impossible to maintain strict adherence to these schedules, a major exercise in restructuring the data base was undertaken to ensure that no seasonal bias had been introduced.

The existence of such a meticulously structured data base afforded an ideal opportunity to assess the extent to which morbidity presented to and care offered by general practitioners (GPs) are seasonally influenced, and therefore the weight that should be attached to this factor in the design of related studies.

\section{Methodology}

The study of the process of care involved 208 GPs from Manchester, Salford, and Trafford and 24 GPs from adjacent affluent suburbs (58\% of all GPs invited to participate). The extent to which those participating were representative of all study area GPs, the reliability of the data, and the overall design and methodology of the study are reported in detail elsewhere. ${ }^{12}$ Broadly, apart from a slight bias towards younger GPs, there was little to distinguish those who participated from all GPs in the area, when a range of known personal and practice characteristics were compared. Reliability was assessed by comparing information recorded on the standard encounter form (available from the authors) by a randomly selected group of GPs with that obtained from their patients by independent raters. The level of agreement between GPs and interviewers ranged from $81 \%$ to $99 \%$, the majority of the data achieving agreement in excess of $96 \%$.

Participants were asked to provide information on all consultations taking place on each of a sample of 20 days, structured to balance days of the week and quarters of the year. However, several of the original 90 
period due to practice or personal problems, and for an appreciable number completion was achieved only by extending the data collection period, which resulted in duplication of a particular quarter in two calendar years. Additionally, those doctors who worked less than five weekdays were over-represented by the 20 days. It was apparent that to overcome these problems the data would have to be pruned quite heavily, and advice from clinicians was sought on how most effectively to preserve seasonal balance with a reduced number of recording days from each GP. There was broad agreement that although seasonal data were commonly reported in quarters, there was no clinical foundation for this, and therefore no reason why recording dates could not be selected to represent one full working week in each of three four-month periods. Further discussion refined this to three periods most likely to highlight any seasonal shifts in morbidity: February-May; June-September; October-January.

There were 31 GPs excluded from the new data base through failure to record on a sufficient spread of days. The majority of the remaining 201 GPs each contributed five weekdays per four-month period, an overall average of 234 recording days per month. All months were therefore weighted to this standard in subsequent analysis.

\section{Results}

NUMBERS OF CONSULTATIONS

Overall, while monthly fluctuations in the numbers of consultations returned were common, there was no evidence of a seasonal pattern. For instance, December produced the lowest standardised number of consultations (6119) while January emerged as the peak month of the general practice year (7530). Even the extent of these monthly shifts should not be overemphasised. In terms of the number of patients an "average" GP sees in one day, the range is not great (26 per day in December to 32 per day in January). The stereotype of a consultation load gathering momentum in the autumn, increasing to a mid-winter peak, and slowly falling thereafter is not supported by these data. A plot of the cumulative distribution of consultations throughout the year wholly supports the null hypothesis that there is no seasonal variation (fig 1 ).

\section{PATIENT CHARACTERISTICS}

Age, sex, and social class of consulting patients were examined for evidence of monthly/seasonal variations in the proportion of attenders within each category. No marked variations were found.

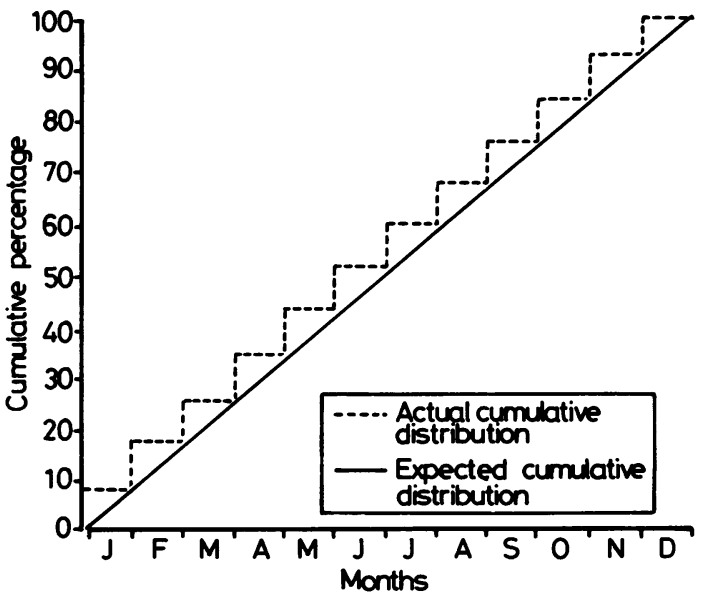

Fig 1 Cumulative distribution of number of consultations and that expected under hypothesis of no seasonal distribution (corrected for number of days recorded).

\section{CONSULTATION CHARACTERISTICS \\ Site}

Home visits accounted for $11 \cdot 7 \%$ of all consultations. There is reason to suspect that there was considerable under-reporting of home visits, but no reason to believe that this was more prevalent at particular times of the year. The winter months produced the highest proportion of home visits, as might be expected. It has been postulated that GPs took the opportunity to 'catch up' on their visits to the elderly and chronically sick during relatively quiet periods, but it is difficult to find evidence to support this. The two peak months for home visiting were January (14\% of consultations) and December (13.9\%), these months already having been shown to have the highest and the lowest numbers of consultations respectively. The pattern is set out in the table.

Site of consultation and new/old ratios

\begin{tabular}{lll}
\hline Time period & $\begin{array}{l}\text { Site } \\
\text { \%ohome visits }\end{array}$ & New/old ratio \\
\hline January & $14 \cdot 0$ & $60: 40$ \\
February & $12 \cdot 6$ & $56: 44$ \\
March & $11 \cdot 6$ & $54: 46$ \\
April & $10 \cdot 4$ & $55: 45$ \\
May & $11 \cdot 6$ & $58: 42$ \\
June & $10 \cdot 5$ & $56: 44$ \\
July & $11 \cdot 8$ & $54: 46$ \\
August & $11 \cdot 4$ & $53: 47$ \\
September & $10 \cdot 4$ & $56: 44$ \\
October & $10 \cdot 8$ & $54: 46$ \\
November & $11 \cdot 3$ & $53: 47$ \\
December & 13.9 & $55: 45$ \\
& & $55: 45$ \\
Overall & $11 \cdot 7$ & \\
\hline
\end{tabular}


New/Old

The proportion of patient-initiated ('new') consultations compared with doctor-initiated ('old') consultations remained relatively stable throughout the year. Only January and, to a lesser extent, May showed increased new to old ratios.

\section{Diagnoses}

Only one ICD diagnostic category showed seasonal fluctuations of any magnitude, and that was predictably 'respiratory conditions'. This category accounted for $17.7 \%$ of all first diagnoses made, ranging from $12.4 \%$ in August to $23.1 \%$ in January. Figure 2 shows the distribution of respiratory cases throughout the year. At a more detailed level of diagnostic classification, a small number of diagnostic groups outside the respiratory category showed some variation. Two of these, unspecified viral $(1 \cdot 3 \%$ of all diagnoses) and otitis media $(1.4 \%)$, could be seen as closely related to respiratory tract infections and showed similar seasonal patterns. Childhood exanthems $(0 \cdot 7 \%)$ and rash/urticaria/pruritus $(0 \cdot 8 \%)$ also demonstrated small, unsurprising shifts. Obesity $(0.7 \%)$ was the only other condition accounting for more than $0.5 \%$ of diagnoses and exhibiting seasonal variation (in this case a marked reduction in presentations in November to January).

\section{Prescribing, tests, and referrals}

Throughout the study period, a prescription was issued in $71 \%$ of cases. Monthly rates varied from $69 \%$ to $73 \%$ and there were no discernible seasonal variations. The same held true for laboratory tests (mean $4 \cdot 1 \%$, range $3 \cdot 4-4 \cdot 6 \%$ ), $x$ rays (mean $1 \cdot 7 \%$, range $1.4-1.9 \%$ ), referrals to consultant (mean $6 \cdot 1 \%$, range $5 \cdot 6-6 \cdot 5 \%$ ), and other referrals (mean $1 \cdot 8 \%$, range $1 \cdot 3-2 \cdot 1 \%)$. Such small variations as did

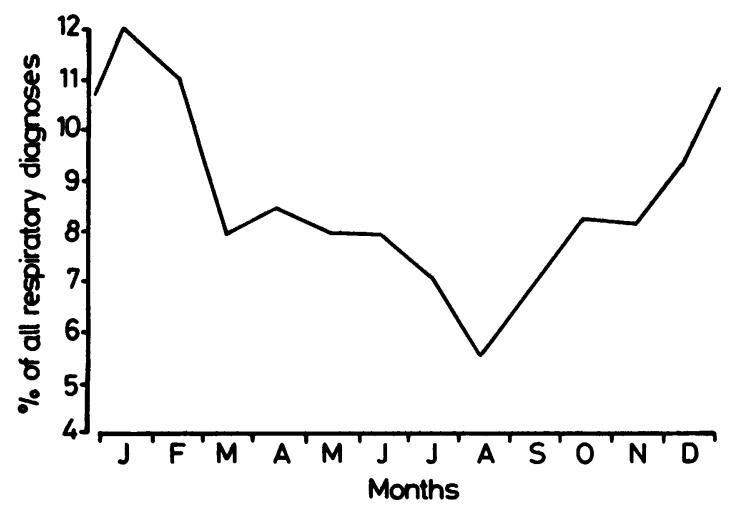

Fig 2 Annual distribution of respiratory diagnoses (corrected for number of days recorded). exist were not related to the actual number of cases seen in a month; there was no evidence that reduced consultation numbers led to increased "activity", that high numbers imposed constraints, or that the reverse held true.

\section{Discussion and Conclusions}

There is a growing volume of literature seeking to examine seasonal patterns in the incidence and prevalence of certain medical conditions and describing statistical tests appropriate to such an exercise. ${ }^{34}$ Particular attention has been paid to schizophrenia, suicide and para-suicide, and conception and birth rates, while individual studies have investigated ischaemic heart disease ${ }^{5}$ ulcerative colitis, ${ }^{6}$ sudden infant death syndrome,${ }^{7}$ peptic ulcer perforation, ${ }^{8}$ and many more. Obviously, while interesting in themselves, the impact made by these conditions on general practitioner services is slight in terms of total consultation numbers. Of more relevance to primary care are seasonal variations in the presentation of more common complaints, where respiratory tract infections, measles, and allergic conditions have attracted attention..$^{9-12}$

Although it is difficult to assess the extent to which perceptions of fluctuating workload have been influenced by the literature on seasonality, it is apparent that there exists in general practice widespread acceptance that seasonal variations are important and must be considered when designing studies, despite any problems this might create. The Second National Morbidity Study ${ }^{13}$ placed the heavy burden of daily recording over a full year on its contributors, which may have restricted the number and range of GPs prepared to take part. Richardson and his colleagues ${ }^{14}$ found it necessary to restrict the amount of information collected in their year-long study of consultations in north-east Scotland. Williams' study $^{\mathbf{1 5}}$ of workload in south Wales consisted of recording periods in each quarter of the year "so that the four seasons were represented". The data base on which this paper draws represented a major investment in time, manpower and resources over a period of 18 months, during which every effort was made to ensure that a true picture of annual consultation patterns in an urban area was obtained, both by recruiting as many general practitioners as possible and by carefully scheduling their recording dates to eliminate possible seasonal bias.

Subsequent analyses have indicated that remarkably little overall seasonal variation exists. It is true that total numbers of consultations do show considerable fluctuation from month to month, but these fluctuations do not form any seasonal patterns and may not in themselves be stable throughout 
successive years. Nor is there any evidence to suggest that the characteristics of patients consulting and the pattern of care they receive varies seasonally; only in terms of the proportion of home visits could seasonal variation be detected in the form of the consultation.

Examination of the clinical content of consultations revealed only one area that could pose serious problems of seasonal bias. Not unexpectedly, proportionately fewer respiratory disorders were seen in the summer months, and the type of respiratory problem seen during the remainder of the year also varied. Thus, with the advent of spring, falling numbers of upper respiratory tract infections, bronchitis, and influenza were bolstered by increasing numbers of hayfever cases. No influenza epidemics occurred during the study period, and thus even greater seasonal imbalance in respiratory complaints could result in an epidemic year. Whether this would be sufficient to induce a seasonal peak in the total number of consultations is debatable, particularly in view of seemingly increased public willingness to treat themselves in self-limiting conditions, coupled to the levelling effect of a rising proportion of elderly patients suffering from chronic, degenerative rather than infectious diseases. It is certainly interesting to note that the proportion of diagnoses falling into each ICD category remained virtually unchanged by the 'culling' of consultations from the heavily overrepresented months of July to November. The restructuring failed to change the balance by so much as a single percentage point, even for respiratory disorders, which remained at $18 \%$ of all first diagnoses.

It seems that some reappraisal is necessary of the part that supposed seasonal variation should play in the design of research studies in general practice. Where the aims of a study include a description of comparative morbidity, it would no doubt be prudent to consider a data collection period of some months' duration, and certainly any study specifically concerned with respiratory disorders should take account of the seasonal nature of such complaints. It appears, however, that the great majority of the content of general practice is not seasonally influenced. Audits of care for non-respiratory conditions, investigations of prescribing, referral, and laboratory usage might well be mounted over reduced timescales. Utilisation patterns among patients of different age groups and social classes could also be viewed over a shorter period of time.

Conversely, wide variations in workload and patterns of care exist among individual GPs. There are extensive differences in the use of hospital-based services and reliance on prescribed medication. In addition, while nearly half of the GPs involved in this study saw patients in relatively constant numbers throughout the year, for the remainder differences did exist but were balanced out over the large number of doctors involved. This suggests that efforts and resources must continue to be devoted to recruiting a large, representative sample of general practitioners to any study attempting to establish a comprehensive picture of service provision or workload.

The data on which this paper draws represent a considerable investment of time, effort, and goodwill by 234 general practitioners in the Greater Manchester area. Processing these data has involved a dedicated support team of eight members of the DHSS Research Unit, Department of General Practice, University of Manchester. Grateful thanks are extended to them, to colleagues who offered advice, and to the DHSS who provided financial support.

\section{References}

${ }^{1}$ Metcalfe D, et al. A study of the 'process' of care in urban general practice. First report for the Department of Health and Social Security. Manchester: DHSS Primary Care Research Unit, Department of General Practice, University of Manchester, 1983.

2 Wilkin D et al. Area variations in the process of care in urban general practice. $\mathrm{Br} \mathrm{Med} J$ 1984; 289: 229-32.

${ }^{3}$ Freedman LS. The use of a Kolmogorov-Smirnov type statistic in testing hypotheses about seasonal variation. $J$ Epidemiol Community Health 1979; 33: 223-8.

${ }^{4}$ Walter SD. The power of a test for seasonality. Br J Prev Soc Med 1977; 31: 137-40.

${ }^{5}$ Rose G. Cold weather and ischaemic heart disease. $\mathrm{Br} J$ Prev Soc Med 1966; 20: 97-101.

6. Cave DR, Freedman LS. Seasonal variation in the clinical presentation of Crohn's disease and ulcerative colitis. Int J Epidemiol 1975; 4: 317-20.

${ }^{7}$ Beal S. Seasonal variation in sudden infant death syndrome. Lancet 1978; i: 1257-9.

${ }^{8}$ Mackay C. Perforated peptic ulcer in the West of Scotland: a survey of 5243 cases during 1954-1963. Br Med J 1966; 1: 701-5.

${ }^{9}$ Symposium. Winter ailments. Practitioner 1979: 223 (1338): 751-91.

${ }^{10}$ Hope-Simpson RE. The role of season in the epidemiology of influenza. J Hyg (London) 1981; 86: 35-47.

${ }^{11}$ Murray $\mathrm{AB}$, et al. The seasonal variation of allergic respiratory symptoms induced by house dust mites. Ann Allergy 1980; 45: 347-50.

${ }^{12}$ Fine PEM, Clarkson JA. Measles in England and Wales. 1. An analysis of factors underlying seasonal patterns. Int J Epidemiol 1982; 11: 5-14.

${ }^{13}$ Royal College of General Practitioners \& Office of Population Census and Surveys. Morbidity Statistics from General Practice, Second National Study 1970-71. Studies on Medical and Population Subjects, No. 26. London: HMSO, 1974.

${ }^{14}$ Richardson IM, et al. A study of general-practitioner consultations in North-East Scotland. J Roy Coll GP 1973; 23: 132-42.

${ }^{15}$ Williams WO. A study of general practitioners' work load in South Wales 1965-6. Reports from General Practice No. XII. London: Royal College of General Practitioners, 1970. 